\title{
Saturation front evolution for liquid infiltration into a gas filled porous medium with counter-current flow
}

\author{
Peter D. Hicks ${ }^{\mathrm{a}, *}$, Mark J. Cooker ${ }^{\mathrm{b}}$, Adrian J. Matthews ${ }^{\mathrm{c}, \mathrm{b}}$ \\ a School of Engineering, Fraser Noble Building, King's College, University of Aberdeen, Aberdeen, AB24 3UE, UK \\ ${ }^{\mathrm{b}}$ School of Mathematics, University of East Anglia, Norwich, NR4 7TJ, UK \\ ${ }^{\mathrm{c}}$ School of Environmental Sciences, University of East Anglia, Norwich, NR4 7TJ, UK
}

\section{A R T I C L E I N F O}

\section{Article history:}

Received 18 June 2013

Received in revised form

9 September 2013

Accepted 14 September 2013

Available online 27 September 2013

\section{Keywords:}

Porous media

Infiltration

Counter-current flow

Compressible gas

\begin{abstract}
A B S T R A C T
The infiltration of liquid into a gas saturated porous network is investigated. Particular attention is paid to the situation in which a pressure gradient in the porous medium drives a gas flow upwards, while a more dense liquid infiltrates down into the reservoir due to gravity. There are two flows in opposite directions. A model is proposed, based upon a compressible gas phase and an incompressible liquid phase. The volume fluxes in each phase are assumed to be governed by Darcy type flow laws, modified to include the permeability caused by both the solid matrix and the impeding of the gas flow by the liquid phase. Isothermal flows are examined in the absence of phase changes. The proposed model is an extension of the traditional Buckley-Leverett model and is used to consider a variety of flows, including carbon sequestration in a porous medium below the seabed and rainfall infiltration into a lava dome.
\end{abstract}

(c) 2013 Elsevier Masson SAS. All rights reserved.

\section{Introduction}

Liquid infiltration into a gas filled porous medium will be investigated. A background pressure gradient will be applied across the porous medium, which drives the gas upwards. The imposed pressure gradient will be insufficient to overcome the effect of gravity on the heavier liquid, setting up counter-current fluid flows as the liquid descends and the gas rises. The effect of the pressure gradient on the gas will be investigated (see Fig. 1).

The infiltration of a fluid into a porous medium is an important process which occurs in many geophysical and industrial situations, including liquid infiltration into soils [1,2], heat exchangers [3,4] and filtration processes [5] as well as biological processes such as fluid flow in the lungs [6,7]. Generally, when such a fluid infiltrates the porous media, it displaces a second fluid that was occupying the void spaces. This displacement can take place by one of two distinct mechanisms. One mechanism is when both fluids completely saturate distinct adjacent regions (on the macroscale) with a mobile interface between the two regions. The second mechanism is that one fluid can displace only a proportion of the second fluid at the macroscale. In this case the fluids co-exist in a state of partial saturation. Of the latter type, Buckley and Leverett [8] began the study of partial saturation of a porous

\footnotetext{
* Corresponding author. Tel.: +4401224 274815.

E-mail addresses: p.hicks@abdn.ac.uk, peterdhicks@yahoo.co.uk (P.D. Hicks).
}

medium and employed the additional assumptions that the process is isothermal and that both fluids are incompressible. The assumption of incompressible fluids is best suited to the case where one liquid is infiltrating a reservoir initially saturated with a second liquid. An example of this process is sea water seeping into an oil reservoir. With these assumptions a single partial differential equation, now called the Buckley-Leverett equation, governs the flow of both fluids. This is a hyperbolic partial differential equation for the saturation of the void-spaces by one of the fluids. Under these theoretical simplifications this equation possesses solutions corresponding to a sharp saturation front which propagates into the porous medium. This analysis has been subsequently reviewed by many authors including Bear and Bachmat [9] and Kaviany [10].

In addition to gravity, capillary suction can have a significant affect on liquid infiltration. Richards [11] investigated the influence capillarity has on liquid infiltration in soils, and the equation derived is now known as Richards' equation. In the derivation of Richards' equation it is assumed that the liquid infiltration is driven by capillarity and gravity, while the second fluid phase occupying the unsaturated void-spaces is largely inert and does not affect the evolution. Richards' equation has been widely studied for its practical applications and also as an example of degenerate parabolic partial differential equations $[12,13]$.

If the problem of liquid infiltrating a pressurized gas-saturated porous medium is investigated, then the assumption of incompressibility may be justified in the liquid. However, due to the pressurization of the reservoir, the gas should be modelled as 

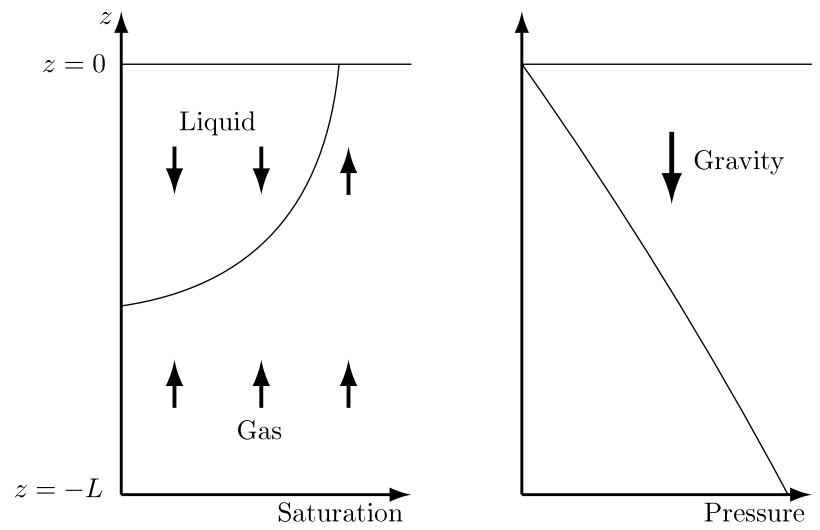

Fig. 1. A schematic diagram of the problem of interest showing liquid infiltrating into a gas filled porous medium. A counter-current flow is established due to phase density differences, gravity and an existing background pressure gradient.

compressible. Therefore generalizations of the models of Buckley and Leverett [8] and Richards [11] are sought, in which one of the fluids present is compressible. Interactions between the different fluid phases occur due to the pressure gradients presented. Our work is motivated by situations in which a liquid is infiltrating a gas-filled porous medium. This situation could arise as the result of carbon sequestration (as part of carbon capture and storage schemes), where pressurized carbon dioxide is stored in an exhausted natural gas field $[14,15]$. Several exhausted gas fields are found under bodies of water (such as the North Sea); it is possible that if the integrity of the reservoir becomes compromised, then seawater may infiltrate the reservoir, releasing carbon dioxide [16,17]. Models of liquid infiltration into porous media are also of relevance to understanding the processes associated with hydraulic fracturing, which attempts to release hydro-carbon based gases trapped within a porous medium by creating new pathways through which the gas can escape by forcing a liquid into the existing pore spaces at very high pressures $[18,19]$.

There has also been recent interest in the interactions of rainfall and volcanic lava domes as the result of a series of dome collapses after periods of intense rainfall at the Soufrière Hills Volcano, Montserrat [20-22]. A high pressure build-up below the surface of the dome, caused by rainwater infiltrating the void spaces of the dome and interacting with the escaping magmatic gases, may contribute to dome collapse [23,24]. In the case of liquid infiltration into both a pressurized porous medium sequestered with carbon dioxide and a lava dome, the pressure gradient across the saturation interface, gravity and the difference in densities between liquid and gas phases may induce counter-current flows, in which liquid falls predominantly due to its weight, whereas the gas is driven upwards by the dominant influence of the pressure gradient. Across many areas of the surface of a lava dome the measured temperatures are high enough to rapidly boil rainwater landing upon it [25]. This continues until sufficient energy has been expended in boiling water to quench the surface temperature to below boiling point $[26,23]$. For temperatures below the boiling point of water there will be a descending saturation front at which the temperature equals the boiling point, and above which the temperature is less than or equal to the boiling point. In this situation boiling will reduce the volume of liquid in the porous medium, slowing the front advancement.

Thermal effects are also prevalent in carbon sequestration, where the Joule-Thomson effect acts to cool the gas as it is forced through a porous medium $[27,28]$. In this case the carbon dioxide may be cooled to the extent that the liquid freezes, which would significantly alter the progress of the front. Carbon dioxide dissolution will occur through interactions between liquid water and gaseous carbon dioxide. The resulting aqueous carbon dioxide solution will increase the liquid acidity and (depending on the rock type) produce porosity and permeability reducing mineral precipitates [29,30]. Additionally, depending on the carbon dioxide concentration and the ambient conditions, the viscosity of an aqueous carbon dioxide solution can differ from that of pure water by up to $38 \%$ [31]. As a preliminary investigation into countercurrent flows driven by a combination of phase density differences, gravity and a background pressure gradient, an idealized problem is considered in a uniformly porous and permeable material, in which all the processes are isothermal and there are no phase changes. However, once our initial model of counter-current flows has been developed, then it can be extended to include these additional effects and be specifically tailored to model either carbon sequestration or rainwater infiltration.

To further simplify the problem, fluid flows will be considered only in one spatial dimension, which will be aligned parallel to gravity. A schematic diagram of the situation of interest is shown in Fig. 1. However, if additional spatial dimensions aligned parallel to the infiltration front are considered, then flows of this type commonly exhibit viscous fingering as an initially planar infiltration front descends and becomes unstable. In the context of rainwater infiltration into a lava dome, the front is unlikely to propagate too far below the surface. Here a one dimensional model is likely to be appropriate as the initially planar front has not had sufficient opportunity to become unstable. In the context of the Buckley-Leverett problem the stability of a planar saturation front has previously been studied by many authors including Tan and Homsy [32], Chikhliwala et al. [33] and Riaz and Tchelepi [34]. However, the stability analysis of the current situation remains an open problem and is worthy of further study.

In Section 2, a system of equations governing the conservation of mass and momentum of a liquid and gas in a partially saturated region is described. Boundary conditions are considered, which naturally give rise to a pressurized upwards gas flow and a counter-current liquid flow. Steady-state solutions and the initial configuration for the gas profile are considered in Section 3, before the resulting system is investigated with and without capillarity in Sections 4 and 5, respectively. Changes to the degree of saturation at the surface and the underlying gas pressure gradient are considered. In the absence of capillarity the regime of small gas pressure gradient is investigated in Section 4.2, and the behaviour simplifies to that which has been previously reported. Finally, Section 6 contains conclusions and analysis resulting from the modelling.

\section{Model development}

We consider liquid infiltration and descent into the void spaces of an initially completely unsaturated gas-filled porous medium. Across the porous medium there is a vertical pressure gradient, which drives an upward gas flow. A system of coordinate axes is chosen, in which the $z$-axis is parallel to the pressure gradient and positive in the upwards direction. The porous medium lies between surfaces at $z=0$ and $z=-H$. Between $z=0$ and $z=$ $-L$ a pressure difference exists and in an initial dry configuration this is denoted by $\left[P_{g}\right]$, with a constant pressure $P_{g, 0}$ at $z=0$ and $L<H$. Attention is restricted to the case in which the two fluid phases are immiscible and phase change between the fluids does not occur.

\subsection{Field equations}

The mass flux of gas per unit cross sectional area of the porous medium $\tilde{\boldsymbol{m}}_{g}$ is then related to the gas volume flux per cross sectional area of void-space $\tilde{\boldsymbol{v}}_{g}$, through the relationship

$\tilde{\boldsymbol{m}}_{g}=\phi \tilde{\rho}_{g}(1-s) \tilde{\boldsymbol{v}}_{g}$ 
where the gas density is denoted by $\tilde{\rho}_{g}$, the porosity $\phi$, and the degree of saturation by the liquid $s$. The saturation is defined to be the proportion of the volume of void-space occupied by the liquid phase. If the gas mass flux into and out of some arbitrary control volume is considered, then an equation governing gas mass conservation can be derived with the form

$\frac{\partial}{\partial \tilde{t}}\left[\phi \tilde{\rho}_{g}(1-s)\right]+\tilde{\nabla} \cdot\left[\phi \tilde{\rho}_{g}(1-s) \tilde{\boldsymbol{v}}_{g}\right]=0$.

If the liquid volume flux per unit cross section area of void-space is denoted by $\boldsymbol{v}_{l}$, then a similar argument leads to an equation for the liquid mass conservation of the form

$$
\frac{\partial}{\partial \tilde{t}}\left[\phi \rho_{l} s\right]+\tilde{\nabla} \cdot\left[\phi \rho_{l} s \tilde{\boldsymbol{v}}_{l}\right]=0
$$

For simplicity, it is assumed that the porosity distribution is homogeneous and liquid is incompressible, making $\phi$ and the liquid density $\rho_{l}$ constant.

We assume that momentum conservation in both the gas and the liquid are adequately described by a modified version of Darcy's law in which a constant, uniform intrinsic permeability $K$, is modified by a relative permeability $K_{g}^{r}$ for the gas phase and $K_{l}^{r}$ for the liquid. The relative permeabilities are assumed to be functions of just the saturation. If $\hat{\boldsymbol{k}}$ is a unit vector in the positive $z$ direction, then the momentum conservations in the gas and the liquid are governed by

$\phi(1-s) \tilde{\boldsymbol{v}}_{g}=-\frac{K K_{g}^{r}(s)}{\mu_{g}}\left(\tilde{\nabla} \tilde{p}_{g}+\tilde{\rho}_{g} g \hat{\boldsymbol{k}}\right)$,

$\phi s \tilde{\boldsymbol{v}}_{l}=-\frac{K K_{l}^{r}(s)}{\mu_{l}}\left(\tilde{\nabla} \tilde{p}_{l}+\rho_{l} g \hat{\boldsymbol{k}}\right)$,

respectively. In these equations $\mu_{g}$ and $\mu_{l}$ are the dynamic viscosity of the gas and liquid phases, respectively, which are assumed constant in each phase. The gas pressure, $\tilde{p}_{g}$, is related to the liquid pressure, $\tilde{p}_{l}$, through the capillary pressure, $\tilde{p}_{c}=\tilde{p}_{g}-\tilde{p}_{l}$. Therefore the liquid pressure can be eliminated from the liquid momentum conservation equation to leave

$\phi s \tilde{\boldsymbol{v}}_{l}=-\frac{K K_{l}^{r}(s)}{\mu_{l}}\left(\tilde{\nabla} \tilde{p}_{g}-\tilde{\nabla} \tilde{p}_{c}+\rho_{l} g \hat{\boldsymbol{k}}\right)$.

The capillary effects contribute to the motion through the term $\tilde{\nabla} \tilde{p}_{c}$, in the liquid momentum conservation equation (5). The capillary pressure is assumed to be a function purely of the saturation and (based on the models of van Genuchten [2]) is taken to be

$\tilde{p}_{c}(s)=\sigma \sqrt{\frac{\phi}{K}} J(s)$,

where $\sigma$ is the surface tension coefficient of the liquid-gas interface, and the non-dimensional van Genuchten [2] J-function has the form

$J(s)=\left(s^{-1 / m}-1\right)^{1 / n}$

where $m=1-1 / n$ and $n>1$ is a fitting parameter, the choice of which depends upon the structure of the porous medium.

In this simplified model there is no momentum transfer between the two fluid phases, owing to the relative movement. In physical problems, the relative permeabilities present in these equations have complicated dependences on many variables, including the degree of saturation, the nature of the rigid matrix, the temperature, and the fluids in question. However, our attention shall be restricted to the simple case in which the relative permeabilities depend solely on the saturation, s. Kaviany [10] provides a number of empirical relationships appropriate for different types of porous matrix and fluids, in particular we use

$K_{g}^{r}(s)=\left(1-s^{2}\right)(1-s)^{2}$,

$K_{l}^{r}(s)=s^{4}$,

which are originally due to Corey [35] and have been used to model permeability in geological formations.

The model is completed by an equation of state for the gas and we take

$\frac{\tilde{p}_{g}}{P_{g, 0}}=\left(\frac{\tilde{\rho}_{g}}{\rho_{g, 0}}\right)^{\gamma}$,

for a reference pressure and density $P_{g, 0}$ and $\rho_{g, 0}$ respectively. Appropriate choices for the constant $\gamma$ are 1 for isothermal gas and 1.4 for adiabatic dry air at $20^{\circ} \mathrm{C}$. For carbon dioxide at $10{ }^{\circ} \mathrm{C}$ involved in carbon sequestration, $\gamma=1.300$ [36] and for the porous lava dome example adiabatic flow of water vapour at $100{ }^{\circ} \mathrm{C}$ corresponds to $\gamma=1.324$. For gases escaping through vents around the lava dome at the Soufrière Hills Volcano, Montserrat the appropriate values are those corresponding to water vapour, as samples taken by [37] were found to be in excess of $90 \%$ water by volume. Other equations of state can readily be considered extending the basic model, while the more complicated extensions to non-isothermal interactions will form the basis of a subsequent paper. If non-isothermal effects were included then it would be necessary to also model evaporation and condensation, between the liquid water and the water vapour phases.

\subsection{Boundary conditions}

The evolution of the pressures and the saturation are investigated, starting from an initially completely dry porous network through which a steady, uniform gas mass flux is driven upwards by the pressure gradient. At the top of a carbon sequestration reservoir, the pressure is assumed to be a constant, $P_{g, 0}$, so the boundary condition is

$\tilde{p}_{g}(z=0, t)=P_{g, 0}$.

The surface of a lava dome is at ground level, where the gas pressure can be fixed at constant atmospheric pressure (although without loss of generality a constant can be added to all the pressures within the model). An idealized liquid inflow condition is envisaged in which the saturation, $s$, jumps from zero at $t=0$ to some positive fixed constant in the range $[0,1)$ for positive time, to allow gas to escape through the surface:

$s(z=0, t)=s_{0} \chi(t)$,

where $\chi(t)$ is the Heaviside function

$\chi(t)= \begin{cases}1, & \text { for } t>0, \\ 0, & \text { otherwise }\end{cases}$

and the constant $s_{0} \in[0,1)$ determines the value of the saturation at the surface. This boundary condition is an idealized representation of the sudden presence of liquid at the top of the porous media, caused perhaps by the presence of rainfall upon the surface, or the opening of a liquid leak from the rock above the porous media.

The length scale for liquid infiltration associated with the phenomena of interest is denoted by $L$, which is much less than the thickness of the porous layer, $H$. Far below the region in which there is a perturbation caused by the liquid infiltration, the gas pressure is assumed to be constant and unaffected by the changes occurring close to the upper surface. The depth of the porous matrix is chosen to be large enough that the solutions are not sensitive to its exact value. In the following examples a choice for the infiltration depth of interest of $L=20 \mathrm{~m}$ has been made. 


\subsection{Non-dimensionalization}

We shall now restrict our analysis to one spatial dimension. In order to investigate counter-current flows we shall assume the pressure gradient and gravity are directed parallel to the $z$-axis. If the pressure gradient and gravity are not parallel, then the cosine of the angle between the two must be sufficiently close to unity to allow the liquid weight term to dominate the gas pressure gradient in the liquid momentum flux. The non-dimensional gas and liquid volume fluxes are denoted by $\boldsymbol{v}_{g}$ and $\boldsymbol{v}_{l}$, respectively and the nondimensional gas mass flux $m_{g}$. In this one spatial dimension, the variables in our model are related to non-dimensional equivalents through

$\tilde{\rho}_{g}=\rho_{g, 0}+\left[\rho_{g}\right] \rho_{g}$,

$\tilde{p}_{g}=P_{g, 0}+\left[P_{g}\right] p_{g}$

$\tilde{p}_{c}=\left[P_{c}\right] p_{c}$,

$\tilde{v}_{g}=V_{g} v_{g}$,

$\tilde{v}_{l}=V_{l} v_{l}$,

$\tilde{z}=L z$,

$\tilde{t}=T t$

Here $\rho_{g}, p_{g}, p_{c}, v_{g}$ and $v_{l}$ are dimensionless functions of position $z$ and time $t$. The saturation $s$, which is the proportion of the voidspace occupied by the liquid phase, is already non-dimensional. The gas pressure difference $\left[P_{g}\right]$ is prescribed, which defines the gas density difference $\left[\rho_{g}\right]$, over a region of interest, of height $L$ metres. The top surface is at constant pressure, which is atmospheric pressure if there is no overlying material. The capillary pressure scale $\left[P_{c}\right]=\sigma \sqrt{\frac{\phi}{K}}$, so that $p_{c}(s)=J(s)$. A time scale $T=L / V_{l}$ is chosen, based on the liquid flux.

Substituting into the equation of state (9), we find

$1+\Pi^{-1} p_{g}=\left(1+\delta^{-1} \rho_{g}\right)^{\gamma}$,

where the ratio of the pressure reference state to the pressure difference across the porous medium is

$\Pi=\frac{P_{g, 0}}{\left[P_{g}\right]}$,

and the corresponding density ratio is defined by

$\delta=\frac{\rho_{g, 0}}{\left[\rho_{g}\right]}$.

In a rigid porous matrix and in the absence of phase changes, non-dimensional forms of the gas and liquid mass conservation equations (2) and (3) are given by

$\frac{\partial}{\partial t}\left[(1-s)\left(\delta+\rho_{g}\right)\right]+\beta \frac{\partial}{\partial z}\left[(1-s)\left(\delta+\rho_{g}\right) v_{g}\right]=0$,

$\frac{\partial s}{\partial t}+\frac{\partial}{\partial z}\left(s v_{l}\right)=0$,

where the ratio of gas to liquid volume fluxes is denoted by $\beta=$ $V_{g} / V_{l}$. The aim is to investigate counter-current flows in which the gas is forced upwards by the pressure gradient (overcoming the effect of gravity on the gas), while the pressure gradient is insufficient to overcome the effect of gravity on drawing down the liquid. These opposing velocities are sustained by the disparity in the fluid densities. Balancing the gas flux with the gas pressure gradient implies $V_{g}=K\left[P_{g}\right] / \phi \mu_{g} L$, and a balance between the liquid flux and gravity implies $V_{l}=K \rho_{l} g / \phi \mu_{l}$. This means

$\beta=\mu_{l}\left[P_{g}\right] / \mu_{g} \rho_{l} g L$, and that the non-dimensional forms of the gas and liquid momentum conservation equations ( $4 a$ ) and (5) are given by

$(1-s) v_{g}=-K_{g}^{r}(s)\left(\frac{\partial p_{g}}{\partial z}+A_{g}\left(\delta+\rho_{g}\right)\right)$,

$s v_{l}=-K_{l}^{r}(s)\left(A_{l}^{-1} \frac{\partial p_{g}}{\partial z}-A_{l}^{-1} C \frac{\partial J}{\partial z}+1\right)$,

where the dimensionless measures of the influence of gravity on the gas and liquid flows are denoted by

$A_{g}=\frac{\left[\rho_{g}\right] g L}{\left[P_{g}\right]}$,

$A_{l}=\frac{\rho_{l} g L}{\left[P_{g}\right]}$,

respectively. For counter-current flow a regime in which $0<A_{g}<$ $1<A_{l}$ is anticipated. In the typical parameter regime of interest (see Section 2.4), $A_{g}$ is small and the role gravity plays on the flow of gas is negligible. Consequently, the terms multiplied by $A_{g}$ are subsequently neglected. The capillary number

$C=\frac{\sigma}{\left[P_{g}\right]} \sqrt{\frac{\phi}{K}}$,

measures the influence of capillarity on the flow, and the capillary pressure is given by (7). In the following examples the $J$-function fitting parameter is taken to be $n=2$, as a representative value for a geophysical porous medium and to illustrate an example of the possible behaviour.

In this spatial direction, the gas mass flux Eq. (1) gives

$m_{g}=\left(\delta+\rho_{g}\right) v_{g}$,

where the non-dimensional mass flux $m_{g}$ is related to the component of the dimensional mass flux $\tilde{m}_{g}$ in the direction of interest, through

$m_{g}=\frac{\tilde{m}_{g} \mu_{g} L}{\left[\rho_{g}\right] K\left[P_{g}\right]}$.

Subsequently, the pressure and density scales (constants $\left[P_{g}\right]$ and $\left[\rho_{g}\right]$, respectively) across the porous medium of length $L$ are chosen so that $m_{g}=1$.

If the volume fluxes are eliminated from the mass conservation equations using the momentum conservation equations, then a system of advection-diffusion equations is formed, which is given by

$\frac{\partial}{\partial t}\left[(1-s)\left(\delta+\rho_{g}\right)\right]=\frac{\mu_{l g}}{A_{l}} \frac{\partial}{\partial z}\left[\left(\delta+\rho_{g}\right) K_{g}^{r}(s) \frac{\partial p_{g}}{\partial z}\right]$,

$\frac{\partial s}{\partial t}=\frac{\partial K_{l}^{r}}{\partial z}(s)+\frac{1}{A_{l}} \frac{\partial}{\partial z}\left[K_{l}^{r}(s)\left(\frac{\partial p_{g}}{\partial z}-C \frac{\partial J}{\partial z}\right)\right]$.

In the gas conservation equations (21a), we have made use of (17b) in order to express $\beta=\mu_{l g} / A_{l}$, where $\mu_{l g}=\mu_{l} / \mu_{g}$.

The gas pressure can be eliminated using the equation of state (12). Initially capillarity shall be neglected by taking $C=0$, and for simplicity the examples illustrated focus on the isothermal $\gamma=1$ case. The dimensional scales for the pressure and density are chosen so that $p_{g}(z=-1, t)=\rho_{g}(z=-1, t)=1$, and therefore $\Pi=\delta$, in this case. With these assumptions, the system of Eqs. (21) becomes

$$
\begin{aligned}
& \frac{\partial}{\partial t}\left[(1-s)\left(\Pi+p_{g}\right)\right]=\frac{\mu_{l g}}{A_{l}} \frac{\partial}{\partial z}\left[\left(\Pi+p_{g}\right) K_{g}^{r}(s) \frac{\partial p_{g}}{\partial z}\right], \\
& \frac{\partial s}{\partial t}=\frac{\partial K_{l}^{r}}{\partial z}(s)+\frac{1}{A_{l}} \frac{\partial}{\partial z}\left[K_{l}^{r}(s) \frac{\partial p_{g}}{\partial z}\right] .
\end{aligned}
$$


Eqs. (22a) and (22b) form a coupled system of non-linear advection-diffusion equations for the saturation $s$, and the nondimensional gas pressure $p_{g}$, which can be used to model the behaviour of liquid infiltration into a gas filled porous reservoir. Steady-state solutions are also possible and these will be investigated in the next section.

\subsection{Parameter estimation}

The gas is driven upwards through a porous medium by a pressure difference. Across the top $L=20 \mathrm{~m}$ of porous medium, the pressure difference is denoted as $\left[P_{g}\right]$. The reference gas pressure $P_{g, 0}=10^{5} \mathrm{~Pa}$ and gas density $\rho_{g, 0}=1 \mathrm{~kg} \mathrm{~m}^{-3}$ are measured at the top of the porous medium. We fix the reference pressure and density as well as the pressure difference across the porous medium, so that (9) determines the difference in gas density across the porous medium. With $\gamma=1$, if we take $\left[P_{g}\right]=5 \times 10^{4} \mathrm{~Pa}$, then $\left[\rho_{g}\right]=0.5 \mathrm{~kg} \mathrm{~m}^{-3}$ and $\Pi=\delta=2$. The length over which this pressure difference occurs is $L=20 \mathrm{~m}$, so that the values of the parameters governing the influence of gravity on the gas are $A_{g}=1.96 \times 10^{-3}$, and for the liquid $A_{l}=3.92$. The surface tension coefficient is taken to be $\sigma=0.072 \mathrm{~N} \mathrm{~m}^{-1}$, and therefore, the capillary number is $C=0.17$, respectively. The description and values of the key non-dimensional numbers are summarized in Table 1.

For the interactions between water and air, $\mu_{l g}=48.7$ and therefore it is expected that the term $\beta=\mu_{l g} / A_{l}$ in the gas conservation equation to be $O(1)$ and should be retained in the analysis. If terms of size $O\left(A_{l}^{-1}\right)$ are neglected, then, for fixed surface saturation, the well known Buckley-Leverett piecewise constant liquid infiltration profiles are recovered [9]. Therefore in order to study the interactions between the gas and the liquid, terms which are $O\left(A_{l}^{-1}\right)$ are retained.

\subsection{Numerical solution technique}

The system of Eqs. (21) or (22) is dominated by the gravitydriven saturation advection term, particularly in the absence of capillarity. Special care must be paid to this term to enable accurate and efficient solution. The numerical solution of (21) or (22) is accomplished through operator splitting to separate advection and diffusion terms in equations.

The advective step evolution is calculated by means of an explicit flux limiter centred scheme using the MinBee limiter. This is second-order accurate (limiting numerical diffusion) except in a region close to the saturation front where a first-order method is employed (to eliminate spurious oscillations in a fully second-order method). The non-linear diffusive step evolution is solved using a second-order Crank-Nicolson scheme coupled to a predictor-corrector iterative scheme to deal with the nonlinearities present in this equation. The resulting scheme has been tested with a variety of grid and time steps and the results are independent of both.

\section{Steady-state profiles}

Our aim is to investigate liquid infiltration into an initially dry porous medium, by perturbing about an initially dry steady-state configuration. Before investigating liquid infiltration, we need to know the initial steady-state flow of the gas, when there is no liquid.

\subsection{Steady-state gas profiles in an unsaturated porous medium}

In an unsaturated configuration, (22b) simplifies dramatically to give $s=0$, for which the effective permeabilities $K_{g}^{r}(0)=1$ and $K_{l}^{r}(0)=0$. Having neglected the effect of gravity on the gas flow, (22a) reduces to

$\frac{\partial p_{g}}{\partial t}=\frac{\partial}{\partial z}\left[\left(\Pi+p_{g}\right) \frac{\partial p_{g}}{\partial z}\right]$.

In steady-state the non-dimensional gas flux through the porous medium must be constant and therefore, due to the choice of the gas flux scale, we have $\left(\delta+\rho_{g}\right) v_{g}=\left(\Pi+p_{g}\right) v_{g}=1$. This means that the steady-state version of $(23)$ contains $\partial p_{g} / \partial t=0$ and the integral with respect to $z$ implies

$\left(\Pi+p_{g}\right) \frac{\mathrm{d} p_{g}}{\mathrm{~d} z}=-1$,

where $p_{g}$ now depends only on $z$, not $t$. At the surface, this firstorder ordinary differential equation must satisfy the condition $p_{g}(0)=0$. Therefore, the steady-state gas pressure profile only depends on the non-dimensional pressure driving parameter $\Pi$, as

$p_{g}(z)=\sqrt{\Pi^{2}-2 z}-\Pi$.

Fig. 2(a) shows gas pressure profiles for $\Pi=0.2,0.5,1.0,2.0$ and 5.0. With $\gamma=1$, the gas pressure is proportional to the gas density and these profiles show that gas is driven upwards through the porous medium with its density (see Eq. (12)) falling as it goes. The non-dimensional pressure driving parameter $\Pi$ as defined by (13a) measures the ratio of the reference pressure relative to the pressure difference across the porous medium. Therefore smaller values of $\Pi$ correspond to a greater pressure (and density) difference across the porous medium. This results in a larger pressure gradient and therefore a larger mass flux is driven up through the porous medium.

Alternatively, if the effect of gravity on the gas is included, then starting from the non-dimensional version of Darcy's law for a single gas phase, Eq. (12) with $\gamma=1$ can be used to eliminate pressure, and gas mass conservation to eliminate the gas volume flux, leaving

$\frac{1}{\Pi+p_{g}}=-\frac{\mathrm{d} \rho_{g}}{\mathrm{~d} z}-A_{g}\left(\Pi+p_{g}\right)$.

Again this first order ordinary differential equation must be solved subject to $p_{g}(0)=0$ at the surface, giving pressure profiles of the form

$p_{g}(z)=\frac{\sqrt{A_{g}\left[\left(1+A_{g} \Pi^{2}\right) \exp \left(-2 A_{g} z\right)-1\right]}}{A_{g}}-\Pi$,

in which $z \leq 0$. In the limit $A_{g} \rightarrow 0$, (26) reduces to (24). Fig. 2(b) shows gas pressure profiles for $\Pi=0.2$ and 5.0, and for the non-dimensional gravity parameter $A_{g}=0.0,0.005$ and 0.01 . This plot shows that the influence of gravity is very small. For $A_{g}=1.96 \times 10^{-3}$ and $\Pi=5$ (as in Section 2.4) there is less than $1 \%$ difference between the pressure profiles with and without the influence of gravity at $z=-2$. Therefore, we shall subsequently neglect terms of $O\left(A_{g}\right)$.

\subsection{Steady-state gas profiles in a porous medium with uniform saturation}

In addition to the steady-state pressure profile in an unsaturated porous medium, it is expedient to calculate steady-state pressure profiles with a constant, uniform saturation throughout the porous medium. In particular, we shall investigate how a fixed gas mass flux per unit area is forced through a porous matrix as the degree of liquid saturation takes different constant values. In subsequent time-dependent calculations (subject to special conditions), the saturation profiles are piecewise constant. It is useful 
Table 1

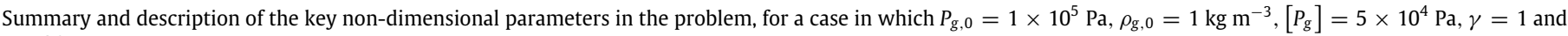
$L=20 \mathrm{~m}$.

\begin{tabular}{|c|c|c|c|}
\hline Parameter & Defined by & Description & Value \\
\hline$A_{g}$ & $(17 a)$ & Ratio of driving pressure to hydrostatic gas pressure & $1.96 \times 10^{-3}$ \\
\hline$A_{l}$ & $(17 b)$ & Ratio of driving pressure to hydrostatic liquid pressure & 3.92 \\
\hline$\beta$ & $(15)$ & Ratio of gas to liquid volume fluxes & 12.4 \\
\hline$\Pi$ & $(13 a)$ & Ratio of upper surface pressure to pressure increase across the porous medium & 2 \\
\hline$\delta$ & $(13 b)$ & Ratio of upper surface density to density increase across the porous medium & 2 \\
\hline C & $(18)$ & Capillary number & 0.17 \\
\hline
\end{tabular}

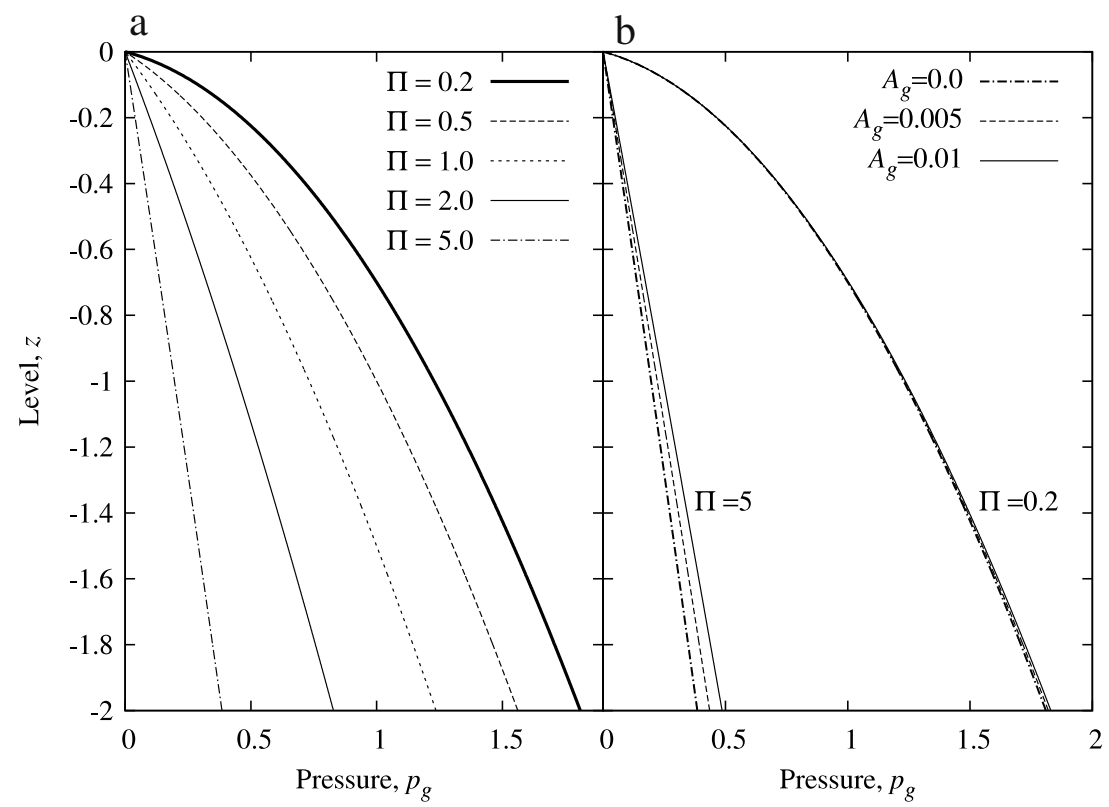

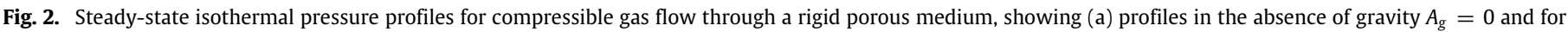
$\Pi=0.2,0.5,1.0,2.0$ and 5.0, and (b) the difference between profiles with and without gravity for $A_{g}=0.0,0.005$ and 0.01 .

to be able to compare these solutions to the steady-state pressure profiles in which the equivalent saturations are constants throughout the porous medium. If we assume $s=\bar{S}$, a constant for all $z$, and the role of gravity in the gas flow is negligible, then the steadystate gas mass conservation equation (22a) reduces to

$\frac{\mathrm{d}}{\mathrm{d} z}\left[\left(\Pi+p_{g}\right) K_{g}^{r}(\bar{S}) \frac{\mathrm{d} p_{g}}{\mathrm{~d} z}\right]=0$.

As a result of the non-dimensionalization, integrating once gives

$$
\left(\Pi+\rho_{g}\right) K_{g}^{r}(\bar{S}) \frac{\mathrm{d} p_{g}}{\mathrm{~d} z}=-1,
$$

a first order ordinary differential equation which generalizes (24) to the case of uniform saturation.

If a rescaled spatial variable $\bar{z}$ is defined satisfying

$$
\bar{z}=\frac{z}{K_{g}^{r}(\bar{s})},
$$

then in terms of the rescaled variable, Eq. (29) becomes

$$
\left(\Pi+p_{g}\right) \frac{\mathrm{d} p_{g}}{\mathrm{~d} \bar{z}}=-1 .
$$

This is a rescaled version of Eq. (24), with solutions similar to Eq. (25)

$p_{g}=\sqrt{\Pi^{2}-2 \bar{z}}-\Pi=\sqrt{\Pi^{2}-\frac{2 z}{K_{g}^{r}(\bar{S})}}-\Pi$.
This illustrates that the steady-state pressure profiles with constant saturation are the same as those without saturation, subject to a rescaling of the spatial variable. Steady-state pressure profiles for a pressure difference $\left[P_{g}\right]=6 \times 10^{4} \mathrm{~Pa}$ and $\delta=5 / 3$ and constant saturation $\bar{S}=0.0,0.1,0.2,0.3,0.4,0.5$ and 0.6 are plotted in Fig. 3. The fact that steady-state pressure profiles with a constant saturation are merely rescaled versions of the steadystate profiles in the absence of saturation should not come as a surprise. In assuming that the momentum conservation in each liquid is governed by a Darcy type law, terms corresponding to interfacial momentum transfer are neglected. The gas flow only depends on the degree to which the liquid impedes the gas flow. Darcy's law predicts the same momentum in the gas whether the liquid phase is stationary, or moving with a constant speed.

\section{Liquid infiltration into a gas saturated reservoir}

If the influence of gravity on the gas flow is neglected, then the only remaining advective term in the system of Eqs. (22) is found in the liquid mass conservation equation and corresponds to the influence of gravity on the liquid phase. Before solving (22) it is worth considering Darcy's law for the liquid phase. In the absence of capillarity, Eq. (16b) in one dimension satisfies

$s v_{l}=-K_{l}^{r}(s)\left(\frac{1}{A_{l}} \frac{\partial p_{g}}{\partial z}+1\right)$.

Hence, if $\frac{\partial p_{g}}{\partial z}>-A_{l}$, then liquid descends into the porous medium. Conversely, if $\frac{\partial p_{g}}{\partial z}<-A_{l}$, then liquid rises in the porous medium. 


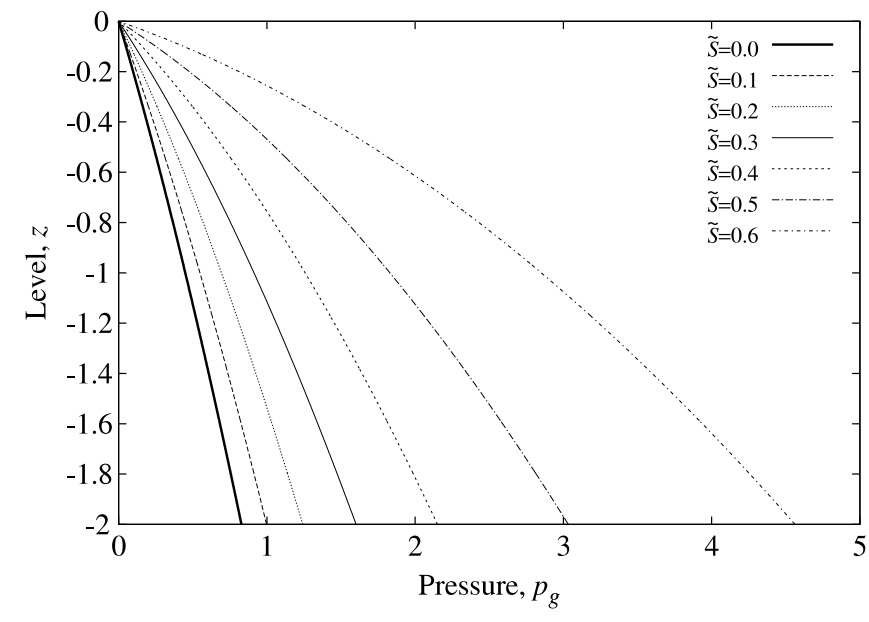

Fig. 3. Steady-state isothermal pressure profiles for compressible gas flow through a rigid porous medium with constant saturations, for $\Pi=2$ and $\bar{S}=0.0,0.1,0.2$, $0.3,0.4,0.5$ and 0.6 .

These conditions relate the driving pressure in the gas phase to the hydrostatic pressure in an equivalent column of liquid.

Fig. 4 shows the time-dependent infiltration of liquid into a porous medium in the absence of capillarity. A regular grid is used, with a grid-spacing $\Delta z=0.001$ and results at integer time-steps after the onset of liquid infiltration. For numerical expediency a saturation pre-layer $s(z, 0)=1 \times 10^{-6}$ is specified below the descending saturation front, to overcome the degeneracy inherent in (22), which occurs when the saturation tends to zero. Tests were conducted with a variety of saturation pre-layer thicknesses and grid-sizes, which showed that the saturation and pressure profiles, and the speed of front infiltration in the numerical results are insensitive to variations of these parameters. Liquid infiltration profiles as plotted in Fig. 4 correspond to a configuration in which initially there is a steady-state gas flux rising through the voidspaces, driven by a pressure gradient $\left[P_{g}\right]=6 \times 10^{4}$ Pa over $L=20 \mathrm{~m}$. Values for the porosity $\phi=0.2$, and permeability $K=$ $1.0 \times 10^{-11} \mathrm{~m}^{2}$ correspond to a typical geophysical rock sample. At the start of the simulation the surface saturation is increased from the pre-layer value to a fixed surface saturation of $s(0, t)=0.4$.

The saturation front moves into the porous medium at a roughly constant speed (Fig. 4(a)). This constant speed is reproduced in all the subsequent experiments in this section and will be analysed later. Where there is liquid water, the degree of saturation $s$ is approximately constant. However, we shall see that variations in the model parameters can change the saturation profiles significantly.

Fig. 4(b) shows the corresponding gas pressure profiles. If the gas is assumed to behave isothermally, then these profiles are proportional to the gas density profiles. Before liquid infiltration the gas profile corresponds to the unsaturated steady profile given by (25) and is indicated in the plot as the thick solid line. To the right of the steady-state profile there are a series of timedependent gas pressure profiles, at integer time-step increments, resulting from the liquid infiltration into the porous medium. These profiles exhibit a cusp which marks the position of the saturation front at that time. Above this cusp the gas profiles are very similar to the steady-state uniform saturation profile, and this corresponds to the fact that for a gas pressure difference $\left[P_{g}\right]=$ $5 \times 10^{4} \mathrm{~Pa}$, the saturation profiles vary only slightly from constant. The gas pressure profiles show an increase above the steadystate profile as the liquid infiltrates the porous medium. This is because liquid infiltration blocks the pathways through which the gas previously escaped and a larger pressure difference is required to drive the gas across the partially saturated region. Fig. 4(b) also shows the steady-state pressure profile corresponding to a uniform saturation throughout the porous medium, $s=0.4$. This steadystate pressure profile is in good agreement with the transient profiles generated above the saturation front, as in this region and for this pressure difference across the porous medium, the saturation decreases only slightly from the value at the surface.

Fig. 5(a) shows the gas volume fluxes and 5(b) the liquid volume fluxes corresponding to the liquid infiltration profiles shown in Fig. 4. Each volume flux is given with respect to the unit cross sectional area of that fluid. In the partially saturated region the gas volume flux increases above the steady-state profile, as more fluid is forced through just the proportion of the void-spaces which are not saturated. This is driven by the steeper pressure gradient evident above the saturation front in Fig. 4(b). Below the saturation front there is a decrease in the gas flow through the void-spaces, which corresponds to the reduction in the pressure gradient seen below the saturation front in Fig. 4(b). The corresponding liquid volume fluxes, shown in Fig. 5, are negative in the partially saturated region, corresponding to the descent of liquid into the porous medium and are zero below the saturation front, where the porous medium is assumed to be completely unsaturated.

We now wish to investigate how the basic time-dependent model described in this section is altered as the result of changes in model parameters. Given viscosities for the liquid and gas phases, the system of Eqs. (22) contains just two non-dimensional numbers: the ratio of the upper surface pressure to the pressure difference across the top $L$ metres of porous medium, $\Pi=P_{g, 0} /\left[P_{g}\right]$ and the parameter $A_{l}=\rho_{l} g L /\left[P_{g}\right]$ measuring the influence of gravity on the liquid flow. We note, therefore, that a decrease in $\Pi$ together with a corresponding proportional decrease in the value of $A_{g}$ physically corresponds to an increase in the pressure difference across the porous medium and a larger counter-current gas flow. This will be investigated in the next section. Independent changes in either $\Pi$ or $A_{g}$ correspond to changes in either the pressure at the top of the porous medium, the vertical length scale or a change in the liquid pressure. Additional significant changes in the flows can be induced by altering the degree of saturation at the top surface and this is investigated in Section 4.3.

There are many other key model parameters which could be investigated when considering counter-current flow in a porous medium, such as the porosity and permeability of the porous medium. However, neither the porosity nor the permeability appear in the non-dimensional numbers contained within the model Eqs. (22) and hence the non-dimensional profiles of the form of Figs. 4 and 5 are unchanged by variations in either porosity or permeability. The porosity and permeability are present in the dimensional volume flux and time scales. This indicates that variation in either of these two parameters would produce different dimensional profiles with smaller volume fluxes and slower liquid infiltration rates corresponding to either decreases in permeability or increases in porosity.

\subsection{Counter-current gas flux variation}

If the pressure difference across the porous medium is increased, this will drive a greater gas flux. This in turn acts against the liquid infiltration and slows the descent of liquid into the porous medium. Fig. 6(a) shows saturation and 6(b) shows pressure profiles for $\left[P_{g}\right]=3 \times 10^{4}, 5 \times 10^{4}$, and $7 \times 10^{4} \mathrm{~Pa}$, with all the remaining parameters the same as in the previous section. The corresponding values of $\Pi$ and $A_{g}$ are given in the figure caption.

In all cases, a liquid front descends into the porous medium at a roughly constant rate. As one would expect, the speed of descent associated with the saturation front is greatest when the pressure gradient driving the counter-current gas flow is least. Small pressure gradients correspond to larger values of $A_{g}$ and 


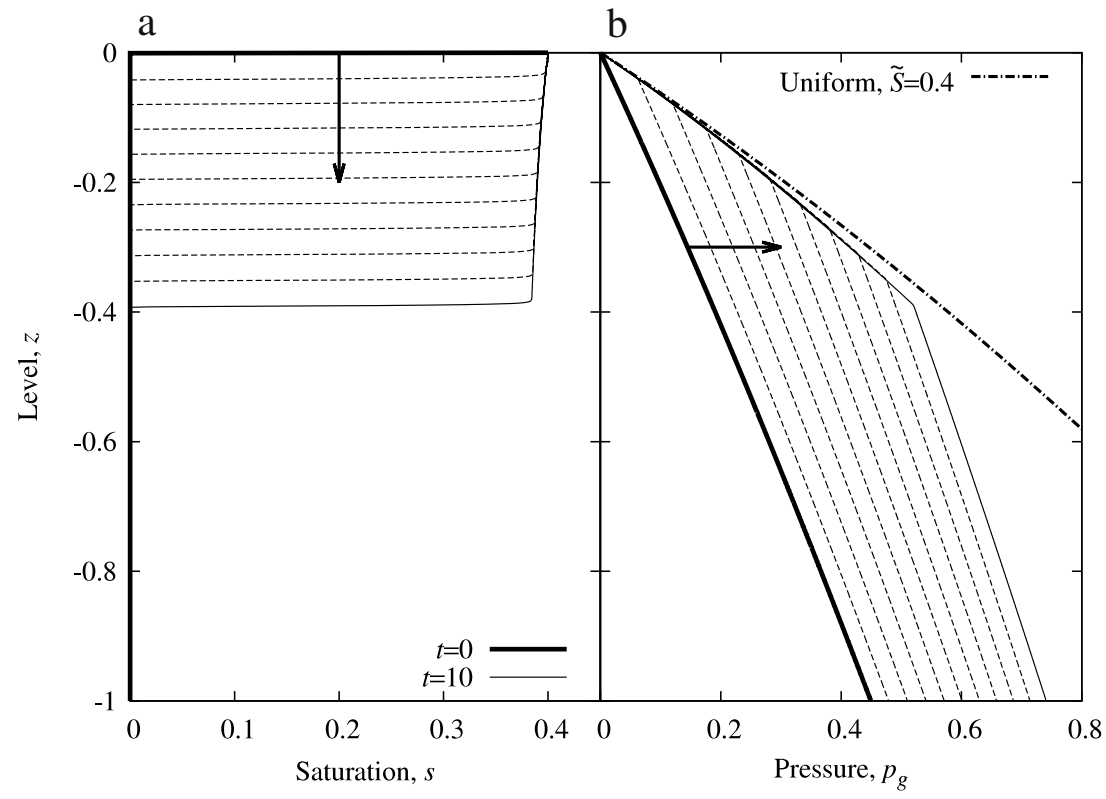

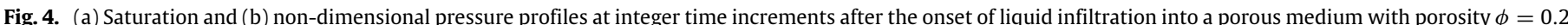

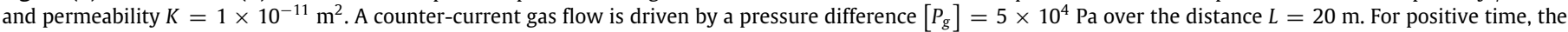
surface saturation $s(0, t)=0.4$. The arrows indicate progress with time. The steady-state pressure profile for a uniform saturation $\bar{S}=0.4$ is also plotted for comparison.

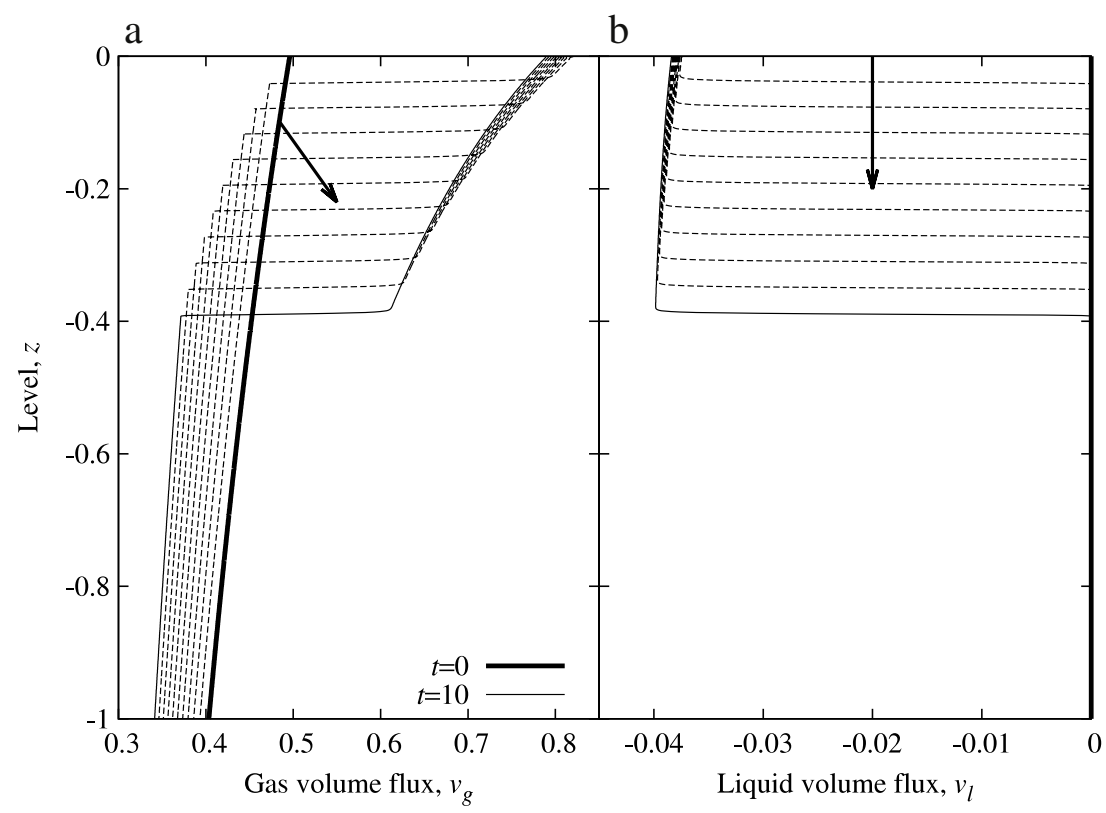

Fig. 5. As Fig. 4, except showing (a) non-dimensional gas volume flux and (b) non-dimensional liquid volume flux. The arrows indicate progress with time.

therefore gravity is even more dominant in the liquid momentum conservation.

A pressure gradient across the porous medium results in a reduction of the degree of saturation as liquid infiltrates the porous medium, with the maximum saturation occurring at the surface. The reduction in the saturation as we descend into the porous medium is most pronounced when there is a large pressure gradient across the porous medium. This is because there is greater competition between the gravity and the gas pressure gradient in the liquid momentum conservation equation, when the gas pressure gradient is largest. For the lowest pressure difference $\left[P_{g}\right]=3.0 \times 10^{4} \mathrm{~Pa}$, the reduction in saturation with depth is barely discernible. As the pressure gradient decreases further, we approach a state in which the gas behaviour has no feedback on the liquid infiltration, which is then purely limited by gravity. This will be investigated further in the next section.

The gas pressure profiles in Fig. 6(b) all show pressure increases when liquid restricts the available void-spaces through which gas can flow. At a given height and time, the maximum gas pressure is the result of a combination of competing factors. As the pressure difference across the porous medium increases the descent of liquid slows. In this case although the steady-state pressure at a given depth might be large, the time taken for liquid to reach that depth may be quite lengthy and the reduction in saturation between the surface and the point of interest may be quite significant, so that the eventual local increase in pressure 

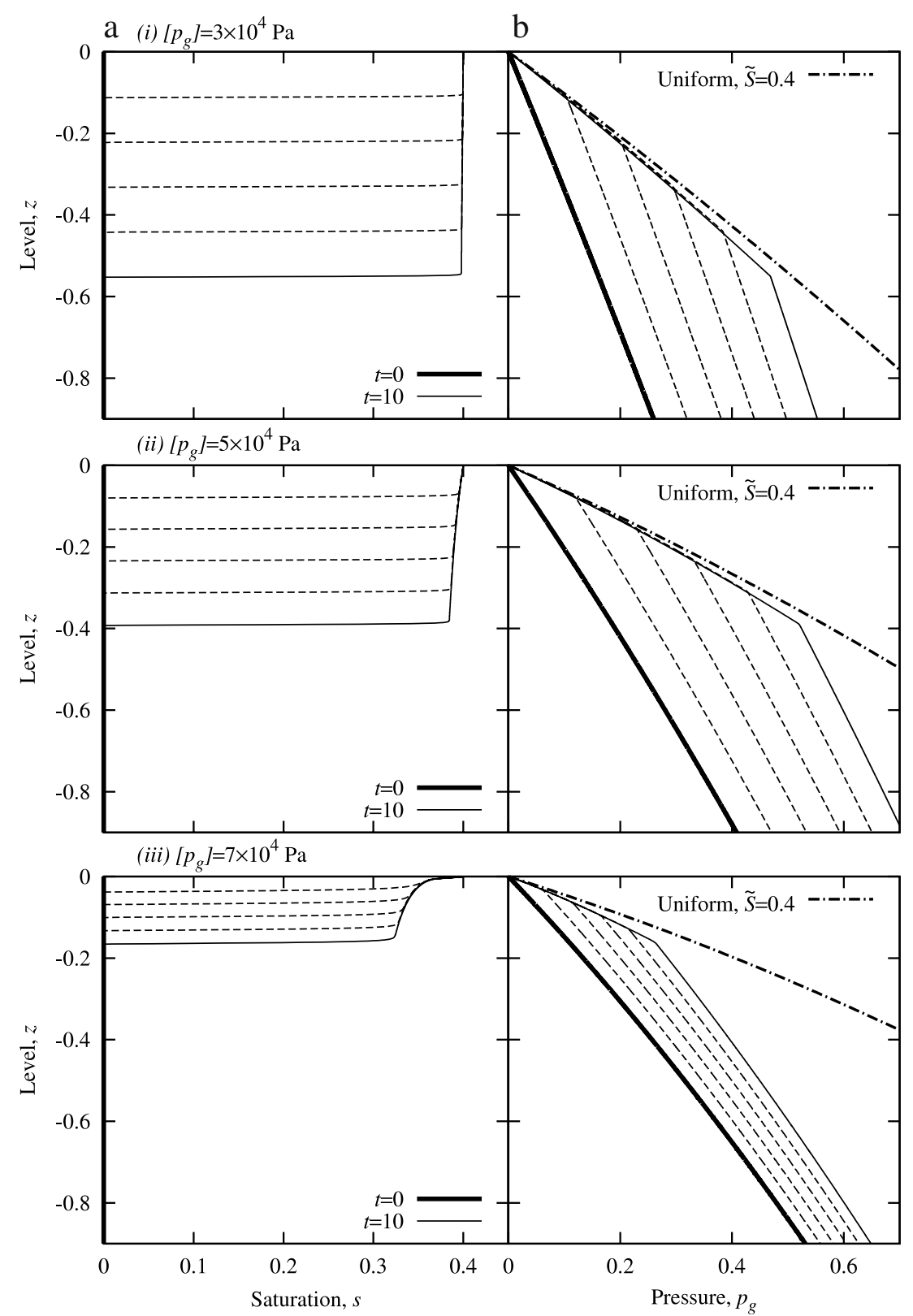

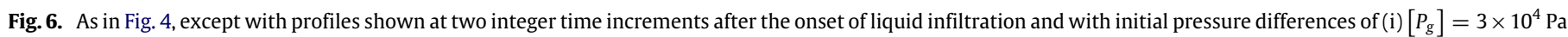

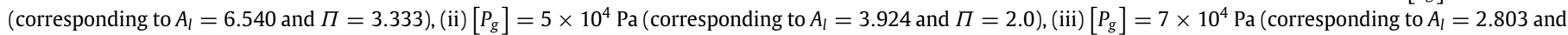
$\Pi=1.429)$.

could be less than in cases with a lower initial pressure difference. Conversely, for a small pressure difference across the porous medium, the liquid may infiltrate faster and maintain a greater proportion of the degree of surface saturation at depth, but the corresponding initial steady-state pressure at a given level is less. Therefore, although a larger increase in the local pressure is possible, the initial local pressure is lower. A determination of the maximum pressure at a given depth therefore depends upon the degree of saturation and the pressure difference across the porous medium.

\subsection{Saturation front position}

Figs. 4 and 6 suggest that the saturation front descends into the porous medium with constant speed, which depends on the counter-current gas mass flux and the degree of surface saturation. The liquid conservation equation (14b) is hyperbolic in some region close to the saturation front. Therefore, if we apply a Rankine-Hugoniot condition across the saturation front, then

$V_{f}=\frac{\mathrm{d} z_{f}}{\mathrm{~d} t}=\frac{\left[s v_{l}\right]_{-}^{+}}{[s]_{-}^{+}}$,

where the position of the front is $z_{f}=z_{f}(t)$, the front velocity is $V_{f}$, and the plus (minus) sign means evaluate above (below) the front. As there is assumed to be zero saturation and liquid volume flux per metre squared below the saturation front, in the absence of capillarity (16b) implies

$V_{f}=\frac{\mathrm{d} z_{f}}{\mathrm{~d} t}=\frac{\left.s v_{l}\right|_{+}}{\left.s\right|_{+}}=-\frac{K_{l}^{r}\left(\left.s\right|_{+}\right)}{\left.s\right|_{+}}\left(\left.A_{l}^{-1} \frac{\partial p_{g}}{\partial z}\right|_{+}+1\right)$,

where the +-sign is the value of property immediately above the saturation front. With the relative permeability of liquid defined as in (8b) and $A_{l} \gg 1$, this implies that the infiltration speed 
of the saturation front has a cubic dependence on the degree of saturation just above the front. The gas pressure gradient is negative throughout the porous medium, in order to drive gas upwards. This means the gas pressure gradient competes with gravity, acting so as to reduce the infiltration of the liquid into the porous medium.

To analyse further the system of Eqs. (22), in many cases of interest we recognize the smallness of the value of the dimensionless parameter $\varepsilon$, defined by

$\varepsilon=\frac{1}{A_{l}}$.

In particular if the liquid phase is water and $L=20 \mathrm{~m}$, then a range of pressure differences from $\left[P_{g}\right]=1.5 \times 10^{4}$ to $5 \times 10^{4}$ Pa corresponds to values of $\varepsilon$ between 0.076 and 0.25 . Further if $\beta=$ $\mu_{g l} / A_{l}=O$ (1), and write $P=\Pi+p_{g}$, then Eqs. (22) can be written

$$
\begin{aligned}
& \frac{\partial}{\partial t}[(1-s) P]=\beta \frac{\partial}{\partial z}\left[P K_{g}^{r}(s) \frac{\partial P}{\partial z}\right], \\
& \frac{\partial s}{\partial t}=\frac{\partial K_{l}^{r}}{\partial z}(s)+\varepsilon \frac{\partial}{\partial z}\left[K_{l}^{r}(s) \frac{\partial P}{\partial z}\right] .
\end{aligned}
$$

We seek $s$ and $P$ as expansions in powers of the small parameter $\varepsilon$ :

$s=s_{0}+\varepsilon s_{1}+\varepsilon^{2} s_{2}+\cdots$,

$P=P_{0}+\varepsilon P_{1}+\varepsilon^{2} P_{2}+\cdots$,

where $s_{i}=s_{i}(z, t)$ and $P_{i}=P_{i}(z, t)$ are coefficients to be determined. This perturbation expansion assumes that the leading order pressure gradient is at most $O(1)$ and we can look for the position of the front in the case of a small pressure gradient across the porous medium.

If the coefficients are matched in increasing powers of $\varepsilon$, then at $O$ (1) we find

$$
\begin{aligned}
& \frac{\partial}{\partial t}\left[\left(1-s_{0}\right) P_{0}\right]=\beta \frac{\partial}{\partial z}\left[P_{0} K_{g}^{r}\left(s_{0}\right) \frac{\partial P_{0}}{\partial z}\right], \\
& \frac{\partial s_{0}}{\partial t}=\frac{\partial K_{l}^{r}}{\partial z}\left(s_{0}\right) .
\end{aligned}
$$

From this equation the leading order solution for $s_{0}$ has the form

$s_{0}= \begin{cases}\bar{S}, & \text { for } z>V_{f} t \\ 0, & \text { for } z<V_{f} t\end{cases}$

Here $\bar{S}$ is a prescribed constant saturation at the surface, $s(0, t)=$ $\bar{S}$, and the velocity of the saturation front, $V_{f}$, is given by the Rankine-Hugoniot condition (35) evaluated with the constant saturation above the front,

$V_{f}=\frac{\mathrm{d} z_{f}}{\mathrm{~d} t}=-\frac{K_{l}^{r}(\bar{S})}{\bar{S}}=-\bar{S}^{3}$.

Integrating this equation, we find that the position of the front satisfies

$z_{f}(t)=-\frac{K_{l}^{r}(\bar{S})}{\bar{S}} t=-\bar{S}^{3} t$,

where the final equality in both (41) and (42) assumes that the relative permeability with respect to the liquid phase is given by (8b). If the counter-current gas pressure gradient is assumed to be small, then (42) gives a maximum depth of liquid infiltration.

In the absence of a pressure gradient at leading order in the liquid mass conservation equation, we next consider the leading order equation for the modified pressure $P_{0}$. If piecewise solutions are sought above and below the saturation front, then porous medium equations in each portion are recovered, satisfying

$$
\begin{aligned}
& \frac{\partial P_{0}}{\partial t}=\frac{\beta K_{g}^{r}(\bar{S})}{(1-\bar{S})} \frac{\partial}{\partial z}\left[P_{0} \frac{\partial P_{0}}{\partial z}\right], \quad \text { for } z>V_{f} t, \\
& \frac{\partial P_{0}}{\partial t}=\beta \frac{\partial}{\partial z}\left[P_{0} \frac{\partial P_{0}}{\partial z}\right], \quad \text { for } z<V_{f} t .
\end{aligned}
$$

At the saturation front $z=V_{f} t$, pressure continuity is also required, which corresponds to

$\left[P_{0}\right]_{-}^{+}=0$.

For convenience, Eqs. (43) are written as

$\frac{\partial P_{0}}{\partial t}=A^{ \pm} \frac{\partial^{2}}{\partial z^{2}}\left(P_{0}^{2}\right)$

where

$A^{+}=\frac{\beta K_{g}^{r}(\bar{S})}{2(1-\bar{S})}, \quad$ for $z>V_{f} t, \quad$ and

$A^{-}=\frac{\beta}{2}, \quad$ for $z<V_{f} t$.

With a variables separable solution of the form $P_{0}(z, t)=$ $\mathcal{P}(z) \mathcal{T}(t)$

$\frac{\mathrm{d} \mathcal{T}}{\mathrm{d} t}=-\lambda^{+} \mathcal{T}^{2}, \quad A^{+} \frac{\mathrm{d}^{2}}{\mathrm{~d} z^{2}}\left(\mathcal{P}^{2}\right)+\lambda^{+} \mathcal{P}=0 ;$

for $z>V_{f} t$,

$\frac{\mathrm{d} \mathcal{T}}{\mathrm{d} t}=-\lambda^{-} \mathcal{T}^{2}, \quad A^{-} \frac{\mathrm{d}^{2}}{\mathrm{~d} z^{2}}\left(\mathcal{P}^{2}\right)+\lambda^{-} \mathcal{P}=0 ;$

for $z<V_{f} t$.

In the small pressure gradient limit, the numerical results of Section 4.1 suggest that the gas pressure profiles are constant in time in the partially liquid saturated region above the cusp. This is equivalent to taking $\lambda^{+}=0$ in (47a). This is a quasi-steady approximation in which the effect of the front evolution on the gas pressure occurs over a much shorter time scale than the time scale associated with the motion of the front itself. Assuming the time scale associated with the pressure evolution below the partially saturated region is the same as the time scale associated with the pressure evolution in the partially saturated region, then this too is also much shorter than the time scale associated with the motion of the front and $\lambda^{-}=\lambda^{+}=0$ in (47b). In this case $\mathcal{P}^{2}$ is a linear function of $z$ both above and below the saturation front, which is consistent with the previously calculated steady-state gas pressure profiles. In the quasi-steady regime the corresponding gas pressure profiles are given by

$p_{g_{0}}=\left\{\begin{array}{l}\sqrt{\Pi^{2}-2 z\left(K_{g}^{r}(\bar{S})\right)^{-1}}-\Pi, \quad \text { for } z>V_{f} t, \\ \sqrt{\Pi^{2}-2\left\{z+V_{f} t\left[\left(K_{g}^{r}(\bar{S})\right)^{-1}-1\right]\right\}}-\Pi, \\ \quad \text { for } z<V_{f} t,\end{array}\right.$

where $p_{g_{0}}=P_{0}-\Pi$ is the leading order pressure corresponding to $P_{0}$. Saturation and gas pressure profiles corresponding to (40), (41) and (48) are plotted in Fig. 7 at time intervals $t=0,2,4$, 6,8 and 10 after the onset of liquid infiltration. The results show good agreement with the numerically computed results of the full problem.

A comparison of the small $\varepsilon$ analytical solutions (given by (a) Eq. (40) and (b) Eq. (48)) and the numerically computed profiles is shown in Fig. 8 for time $t=10$. Profiles are shown for $\varepsilon=0.076$ (corresponding to $A_{l}=13.08$ and $\Pi=6.6667$ ), $\varepsilon=0.153$ (corresponding to $A_{l}=6.540$ and $\Pi=3.3333$ ), and $\varepsilon=0.255$ (corresponding to $A_{l}=3.924$ and $\Pi=2$ ). The best agreement is obtained from the smallest value of $\varepsilon$. 


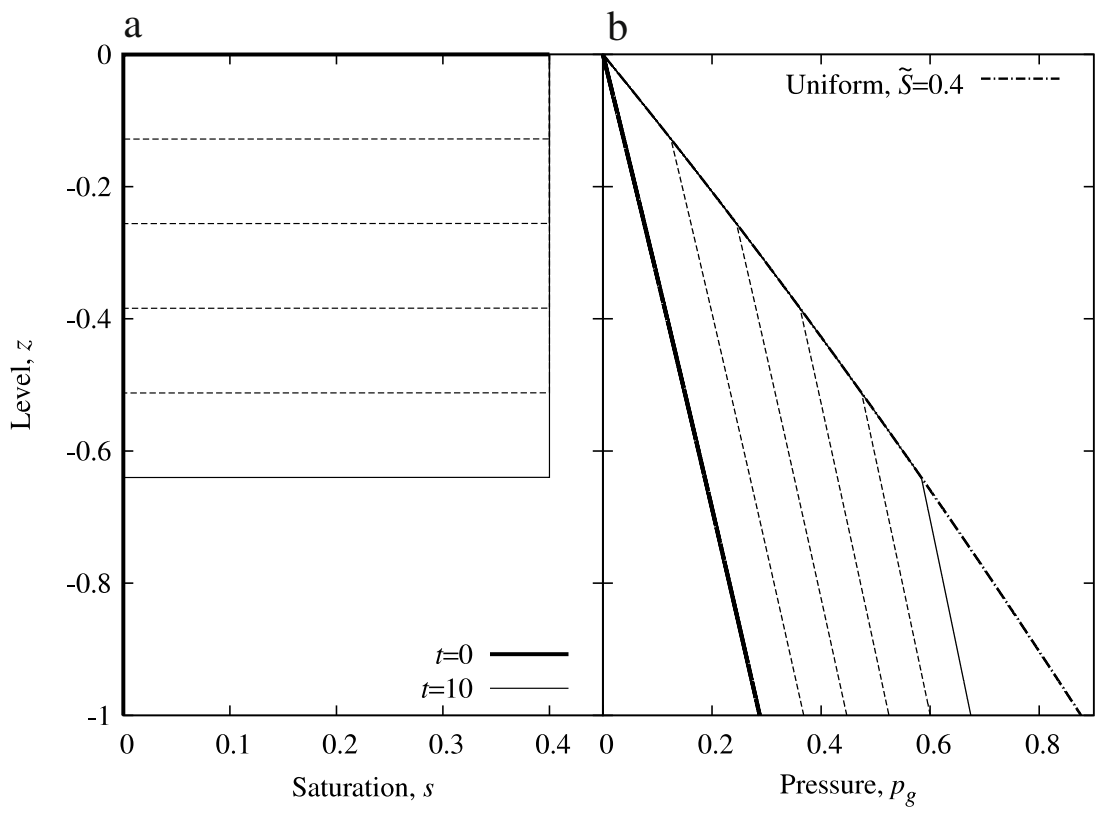

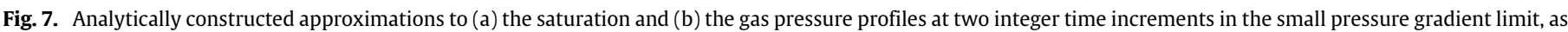

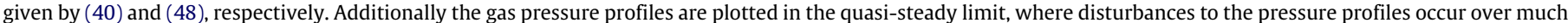
shorter time scales than those associated with the movement of the saturation front.

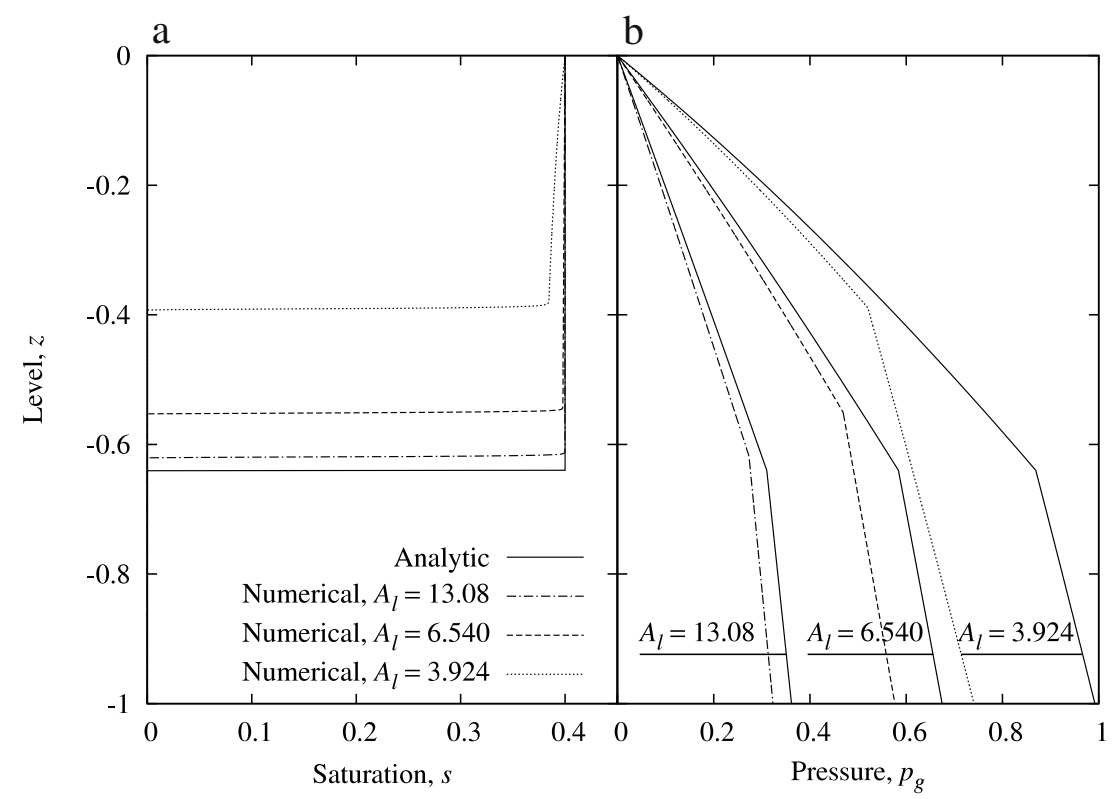

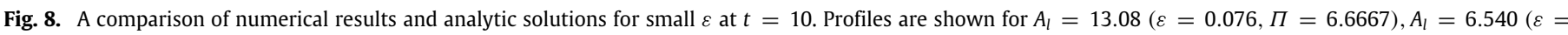
$0.153, \Pi=3.3333)$ and $A_{l}=3.924(\varepsilon=0.255, \Pi=2)$.

\subsection{Surface saturation variation}

If the non-dimensional parameters $\delta$ and $A_{g}$ are held constant, then significantly different model behaviour can be induced by altering the degree of saturation at the surface. Fig. 9 shows the saturation and gas pressure profiles for fixed surface saturations of $s(0, t)=0.2,0.4$, and 0.6 , with all the other parameter the same as in Section 4 . We immediately see that as the degree of surface saturation is increased, the infiltration front descends faster. However, the increase in infiltration speeds, predicted by (42), tails off dramatically as the void-space available for gas flow is reduced and the pressure gradient rises. This reduction in liquid velocity is due to the increase in the pressure gradient in the liquid competing with the gravity-driven liquid infiltration. The competition between gravity and the pressure gradient is most markedly seen when $s(0, t)=0.6$. In this case, the pressure gradient becomes so large that a saturation of $s=0.6$ cannot be maintained in the porous medium and the degree of saturation falls dramatically just below the surface of the porous medium.

The associated gas pressure profiles (shown in Fig. 9(b)) show a cusp at the same depth as the saturation front in all cases. Although not shown for clarity, above the cusp the gas pressure is close to the steady-state pressure profile in the cases in which the saturation profiles are approximately piecewise constant. These cases correspond to the lower values of the surface saturation. With the surface saturation $s(0, t)=0.6$ the deviation of the saturation profile away from a constant profile dictated by the surface saturation is particularly marked. 


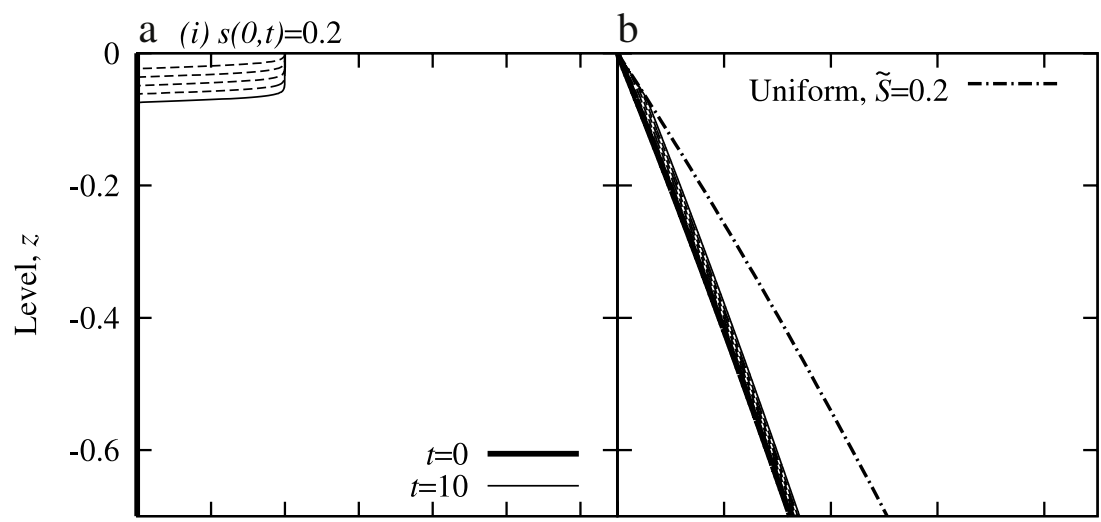

(ii) $s(0, t)=0.4$
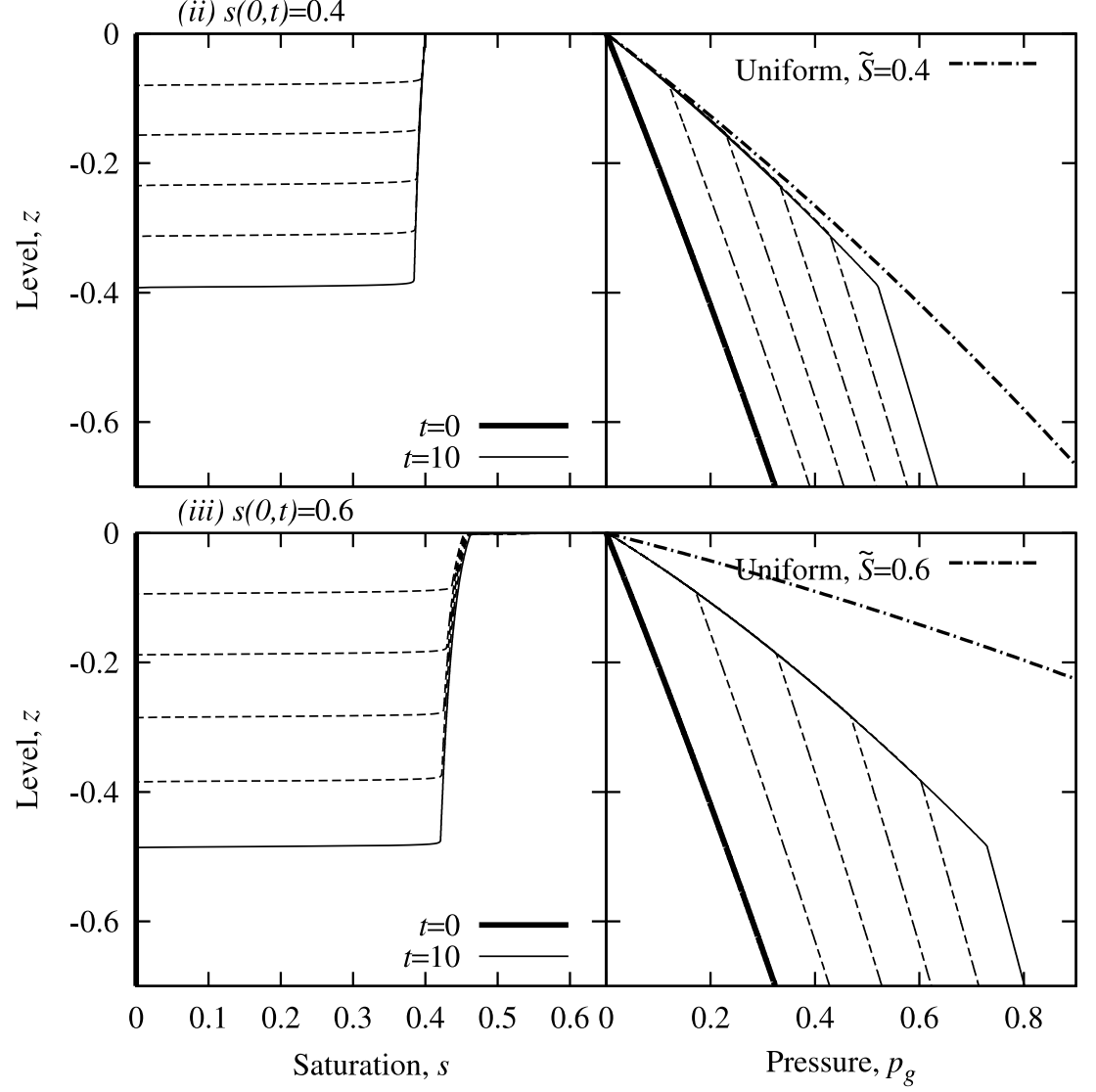

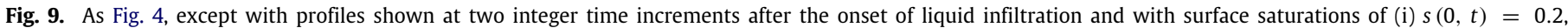
(ii) $s(0, t)=0.4$ and (iii) $s(0, t)=0.6$.

\section{Capillarity}

Until now we have considered the problem of liquid infiltration into a porous medium with a counter-current gas flow in the absence of capillarity. With capillarity added, $C \neq 0$ and the timedependent liquid infiltration is governed by (21). Applying the chain rule to the capillary effect term and assuming that the gas behaves isothermally mean we can write this system of equations as

$$
\begin{aligned}
& \frac{\partial}{\partial t}\left[(1-s)\left(\Pi+p_{g}\right)\right]=\frac{\mu_{l g}}{A_{l}} \frac{\partial}{\partial z}\left[\left(\Pi+p_{g}\right) K_{g}^{r}(s) \frac{\partial p_{g}}{\partial z}\right], \\
& \frac{\partial s}{\partial t}=\frac{\partial K_{l}^{r}}{\partial z}(s)+\frac{1}{A_{l}} \frac{\partial}{\partial z}\left[K_{l}^{r}(s)\left(\frac{\partial p_{g}}{\partial z}-C \frac{\mathrm{d} J}{\mathrm{~d} s} \frac{\partial s}{\partial z}\right)\right],
\end{aligned}
$$

where the capillary pressure equations (6) and (7) imply

$\frac{\mathrm{d} J}{\mathrm{~d} s}=-\frac{1}{n m s^{1+1 / m} J(s)^{n-1}}$, where again $m=1-1 / n$. Taking $n=2$ gives $m=1 / 2$ and

$$
\left.\frac{\mathrm{d} J}{\mathrm{~d} s}\right|_{n=2}=-\frac{1}{\left.s^{3} J(s)\right|_{n=2}},
$$

where $J$ (s) is given by (7). Hence, for $C>0$ the effect of including the capillary pressure, as in (49), is to diffuse the saturation front. To aid numerical computation a thin precursor saturation layer is used below the saturation front to prevent degeneracy.

Fig. 10 shows the same model configuration as Fig. 4, except that capillary effects are added with a value of $C=0.17$. As expected the addition of surface tension diffuses the sharp saturation front in the saturation profiles (Fig. 10(a)). Further increases in $C$ act to diffuse and smooth the front further. Liquid infiltration is also faster, the larger the value of $C$. The corresponding gas pressure profiles are also altered with the sharp cusp at the saturation front now rounded off. 


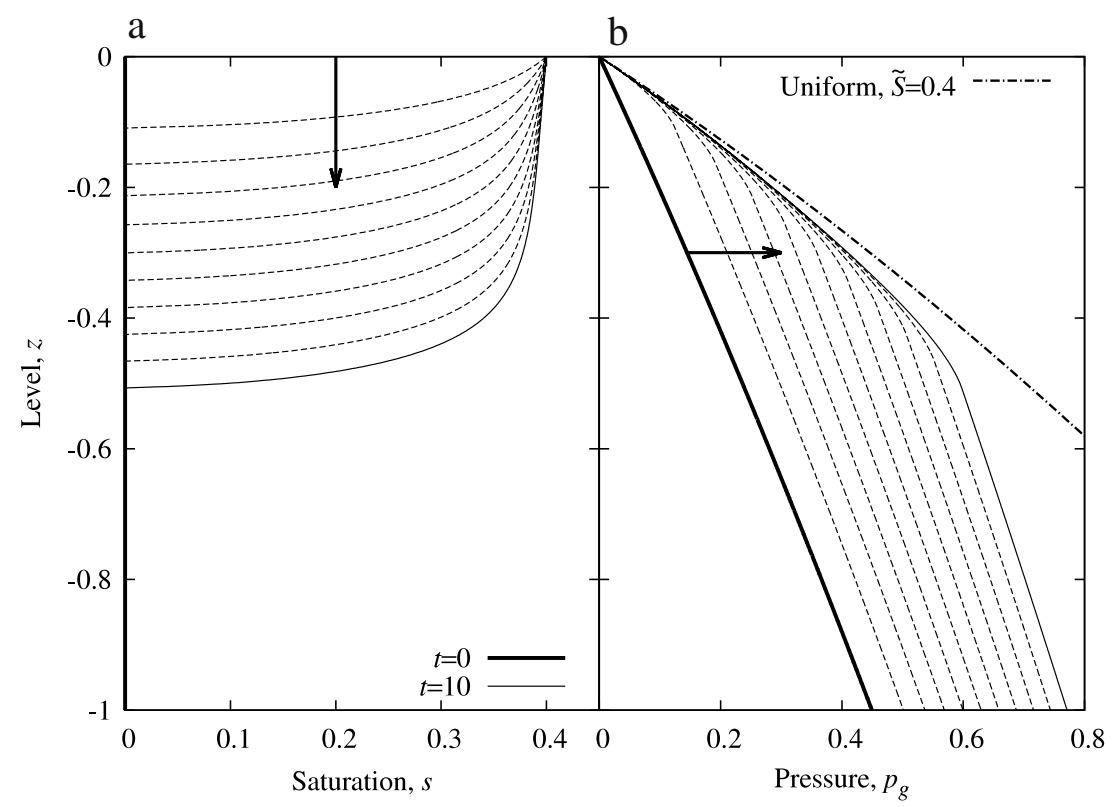

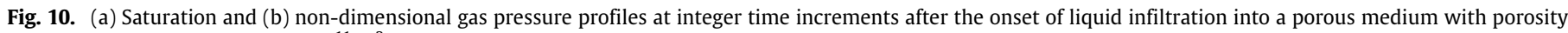
$\phi=0.2$, permeability $K=1 \times 10^{-11} \mathrm{~m}^{2}$ and a capillary number $C=0.17$. The arrows indicate progress with time.

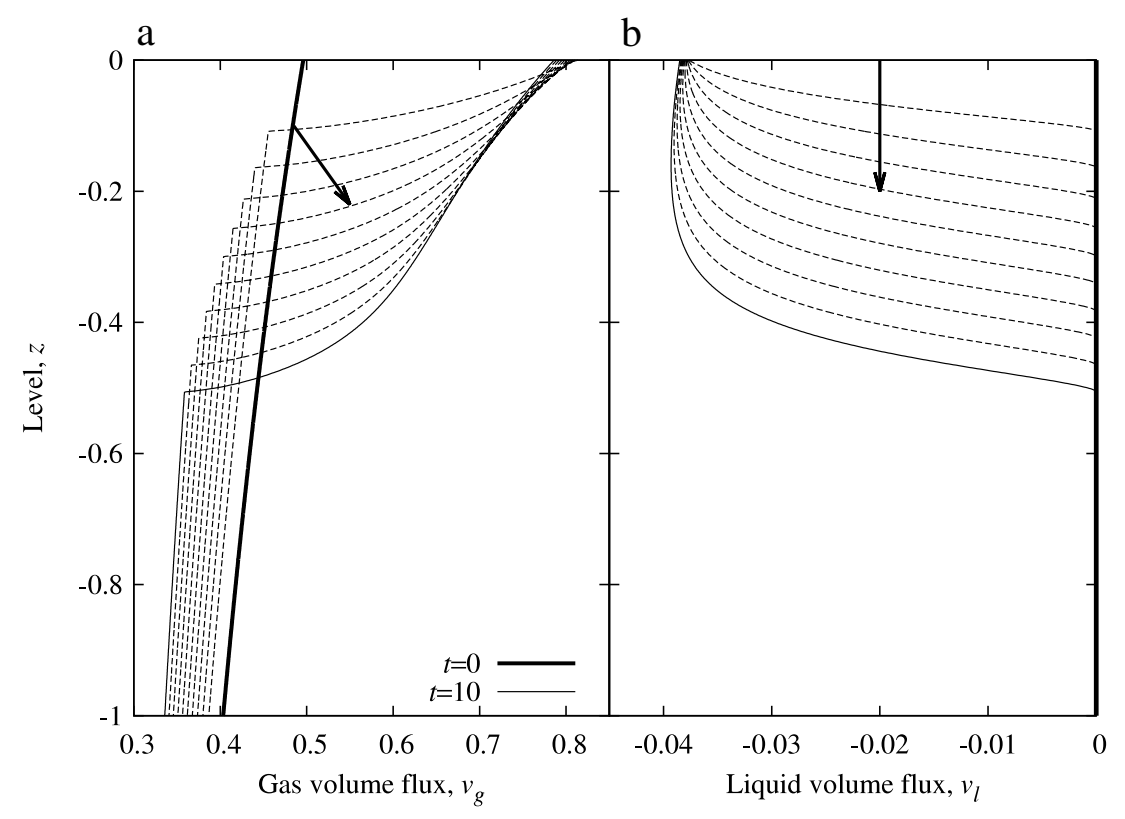

Fig. 11. As Fig. 10, but showing non-dimensional (a) gas volumes and (b) liquid volume fluxes. The arrows indicate progress with time.

Fig. 11(a) shows the corresponding gas volume flux per unit area and 11(b) shows the corresponding liquid volume flux per unit area corresponding to the saturation and gas pressure profiles plotted in Fig. 10. As with the saturation and gas pressure profiles the inclusion of capillarity acts to smooth out the flux profiles, as compared to Fig. 5.

\section{Discussion and conclusions}

The model serves to illustrate the competing processes of a counter-current gas flow on the infiltration of a liquid into an idealized one-dimensional porous medium. Darcy's law with relative permeabilities is used to model the reduction in permeability caused by the presence of the second fluid phase, while the gas is assumed to be compressible. By allowing one of the fluid phases to be compressible extensions to both the Buckley-Leverett and Richards models are created, which exhibit new flow phenomena relevant to a wide range of physically motivated practical problems. The presented analysis incorporates both a background pressure gradient driving the gas upwards, while the density difference allows the liquid to descend through the porous medium.

The key feature of the model is the illustration of the competing processes associated with liquid infiltration and descent driven by gravity and the upwards movement of the gas phase in response to the pressure gradient. Starting from this basic configuration with counter-current liquid and gas flows, perturbations are applied to the basic flow structure and the subsequent evolution investigated. The central observation is that the pressure gradient in the porous medium slows the liquid descent relative to infiltration driven 
purely by gravity. As the pressure gradient rises, the infiltration speed of the liquid falls as the pressure gradient attempts to drive the liquid up and out of the porous medium. Theoretical saturation profiles are found in the small pressure gradient limit, in which the liquid infiltration is governed by gravity.

The degree of saturation is also investigated. The infiltration speed increases with saturation and in the small pressure gradient limit, the infiltration speed is proportional to the cubic power of the saturation just above the interface. As the pressure gradient rises, the liquid infiltration rate again falls (from a maximum speed in the small pressure gradient limit), due to the increased competition between the pressure gradient and gravity. For sufficiently large levels of saturation at the surface, the pressure gradient that is created only allows sufficient liquid to infiltrate the porous medium to saturate the interior of the porous medium at a lower level.

The final modification made to the model is to add capillarity. This acts to diffuse the sharp jump at the lower boundary of the liquid infiltration and generates a more gradual transition in the degree of saturation from a dry porous medium at depth to the partially saturated porous medium just below the surface. Keeping all other conditions the same, the inclusion of capillarity speeds up infiltration.

The infiltration of liquids into gas saturated porous media can have a wider influence than on the changes in the flow structures described in this paper. In a porous medium used for carbon sequestration these results indicate that cracks and defects in an otherwise impermeable boundary to the porous medium could allow water to infiltrate the porous medium and leakage of the stored carbon dioxide. Clearly the escape of carbon dioxide from the reservoir is undesirable, while the pressure increases associated with transient liquid infiltration and gas leakage may result in further increases in the size of the pore spaces through which any leaks occur.

At the Soufrière Hills Volcano, Montserrat, statistical evidence indicates that the probability of lava dome collapse is significantly increased shortly after periods of intense rainfall. For example, the daily probability of lava dome collapse increased from a background value of $1 / 70$ to $1 / 11$ (a sixfold increase), on days when at least $20 \mathrm{~mm}$ of rain fell on the volcano [38]. The process of dome collapse began at the surface within 30 min of the start of the intense rainfall. The proposed mechanism is that after an initial cooling of the dome surface [23], liquid infiltrated into the interior [24], leading to an increase in pore pressure as described here. Subsequently volcanic activity progressed deeper into the volcano, with disturbances still present at a depth of $1 \mathrm{~km}$ after $12 \mathrm{~h}$ [22]. This damaged the porous matrix of the lava dome, ultimately weakening it to the extent that it collapses.

\section{References}

[1] Y. Mualem, Water Resour. Res. 12 (1976) 513-522.

[2] M.T. van Genuchten, Soil Sci. Soc. Am. J. 44 (1980) 892-898.

[3] A. Mohamad, Int. J. Therm. Sci. 42 (2003) 385-395.

[4] B.I. Pavel, A.A. Mohamad, Int. J. Heat Mass Transfer 47 (2004) 4939-4952.

[5] B.Y. Jamaloei, K. Asghari, R. Kharrat, F. Ahmadloo, J. Pet. Sci. Eng. 72 (2010) 251-269.

[6] C.T. DeGroot, A.G. Straatman, in: K. Vafai (Ed.), Proceedings of Porous Media and its Applications in Science, Engineering, and Industry: Fourth International Conference, Potsdam, Germany, 17-22 June, Vol. 1453, AIP, 2012, pp. 69-74

[7] A.F. Miguel, in: J. Delgado (Ed.), Heat and Mass Transfer in Porous Media, in: Advanced Structured Materials, vol. 13, Springer, Berlin, Heidelberg, 2012, pp. 115-137.

[8] S.E. Buckley, M.C. Leverett, Trans. AIME 146 (1942) 107-116.

[9] J. Bear, Y. Bachmat, Introduction to Modeling of Transport Phenomena in Porous Media, Kluwer Academic Publishers, 1991.

[10] M. Kaviany, Principles of Heat Transfer in Porous Media, Springer-Verlag, New York, 1991.

[11] L.A. Richards, Physics 1 (1931) 318-333.

[12] T.P. Witelski, J. Eng. Math. 45 (2003) 379-399.

[13] T.P. Witelski, Transp. Porous Media 27 (1997) 121-134.

[14] E. Lindeberg, Energy Convers. Manage. 38 (1997) S235-S240. Proceedings of the Third International Conference on Carbon Dioxide Removal.

[15] M.L. Szulczewskia, L. Cueto-Felguerosoa, R. Juanes, Energy Procedia 1 (2009) 3421-3428.

[16] D. Silin, T.W. Patzek, S.M. Benson, Int. J. Greenhouse Gas Control 3 (2009) 300-310.

[17] D. Silin, T.W. Patzek, S.M. Benson, Transp. Porous Media 76 (2009) 449-469.

[18] B. Haimson, C. Fairhurst, J. Petrol. Technol. 21 (1969) 811-817.

[19] S.A. Shapiro, C. Dinske, Geophys. Prospect. 57 (2009) 301-310.

[20] A.J. Matthews, J. Barclay, S.A. Carn, G. Thompson, J. Alexander, R.A. Herd, C. Williams, Geophys. Res. Lett. 29 (2002) 1644.

[21] S.A. Carn, R.B. Watts, G. Thompson, G.E. Norton, J. Volcanol. Geotherm. Res. 131 (2004) 241-264

[22] A.J. Matthews, J. Barclay, J.E. Johnstone, J. Volcanol. Geotherm. Res. 184 (2009) $405-415$.

[23] A.J. Matthews, J. Barclay, Geophys. Res. Lett. 31 (2004) L05614.

[24] P.D. Hicks, A.J. Matthews, M.J. Cooker, J. Geophys. Res. 115 (2010) B09212

[25] C. Oppenheimer, P.W. Francis, D.A. Rothery, R.W.T. Carlton, J. Geophys. Res. 98 (1993) 4269-4286.

[26] P.D. Hicks, A.J. Matthews, M.J. Cooker, J. Geophys. Res. 114 (2009) B07201.

[27] K. Pruess, Environ. Geol. 54 (2008) 1677-1686.

[28] A. Singh, G. Baumann, J. Henninges, U.-J. Görke, O. Kolditz, Environ. Earth Sci. 67 (2012) 497-509.

[29] V. Lagneau, A. Pipart, H. Catalette, Oil Gas Sci. Technol.-Rev. IFP 60 (2005) 231-247.

[30] M.J. Mitchell, O.E. Jensen, K.A. Cliffe, M.M. Maroto-Valer, Proc. R. Soc. Lond. Ser. A 466 (2010) 1265-1290.

[31] A.W. Islam, E.S. Carlson, Energy \& Fuels 26 (2012) 5330-5336.

[32] C.T. Tan, G.M. Homsy, Phys. Fluids 29 (1986) 3549-3556.

[33] E.D. Chikhliwala, A.B. Huang, Y.C. Yortsos, Transp. Porous Media 3 (1988) 257-276.

[34] A. Riaz, H.A. Tchelepi, Transp. Porous Media 64 (2006) 315-338.

[35] A.T. Corey, Prod. Monthly 19 (1954) 38-41.

[36] G.W.C. Kaye, T.H. Laby, Tables of Physical \& Chemical Constants: And Some Mathematical Functions, sixteenth ed., Longman Scientific and Technical, 1995.

[37] G. Hammouya, P. Allard, P. Jean-Baptiste, F. Parello, M.P. Semet, S.R. Young, Geophys. Res. Lett. 25 (1998) 3685-3688.

[38] J. Barclay, J.E. Johnstone, A.J. Matthews, J. Volcanol. Geotherm. Res. 150 (2006) 339-358. 